\title{
Self-Assembly of DNA Double-Double Crossover Complexes into High-Density, Doubly-Connected, Planar Structures
}

\author{
Dustin Reishus, Bilal Shaw, Yuriy Brun, Nickolas Chelyapov, Leonard Adleman* \\ Laboratory for Molecular Science, University of Southern California, Los Angeles, CA 90089-2910 \\ E-mail: adleman@usc.edu
}

\section{Supporting Information}

\section{DNA Sequences}

Double-double crossover complex DNA strands (Figures 1 and 2) $\left(5^{\prime}-3^{\prime}\right)$ :

Strand 1: $\quad$ CGAGACCACGTCTTACCTCT

Strand 2: $\quad$ ACGGAAGTGTGAAACTCAGCAGATCCTATGCGTTCGCTTT

Strand 3: GAACGCATAGTCGGCTGGGAAAATGCTTAGAAGATTAAGTTTGATCTCGTGG

Strand 4: TAAGAGCTGAATCATGGATTACTCGGATACGTTAAAGGTAGGGTTTCACACT

Strand 5: CTAGTAGTCCTCCGAGTAATCCATGATCCTACCTTTAACGTATTTGGTAATGGT

Strand 6: GGCTACAGACTGCATTTTCCCAGCCGAAAACTTAATCTTCTAAAATTCTCAAAC

Strand 7: TCTCGACCATTACCAAAGGACGAATTAGTCTGTAGCCAGAGG

Strand 8: TCCGTGTTTGATACTAGAAAGC

Alternative double-double crossover complex DNA strands (Figure 3) (5' - 3'):

Strand 1: GAAAGTGGGAGGTGGAAATGAGTTGA

Strand 2: $\quad$ CTTCTTGGCAGACATTATTAAATTGGTGAGGGCTAC

Strand 3: CCTCACCAGAACGACGACAACATCCGAATAGCAAAACAATATTTAACCTCCCA

Strand 4: TCATTTCCATAATCCATCTTCCTTTTCACGCACCTATATCTCCTAGTCTGCCA

Strand 5: TATGCTTGGTCGTGTGAAAAGGAAGATGGTAGGAGATATAGGTGCGTTGCTAA

Strand 6: TTGCGCTCGATGTTGTCGTCGTTCATTGTTTTGCTATTCGGCTGTTTACGTCT

Strand 7: $\quad$ CTTTCTTAGCAACACGACACAGCGAGCGCAATCAAC

Strand 8: $\quad$ AGAAGAGACGTAACAAGCATAGTAGC

Note that the strands are color-coded and correspond to the colors in Figures 1a and $3 \mathrm{a}$, as well as the folding diagrams. 


\section{Folding Diagrams for the DDX Complexes}

Complexes seen in Figures 1 and 2.

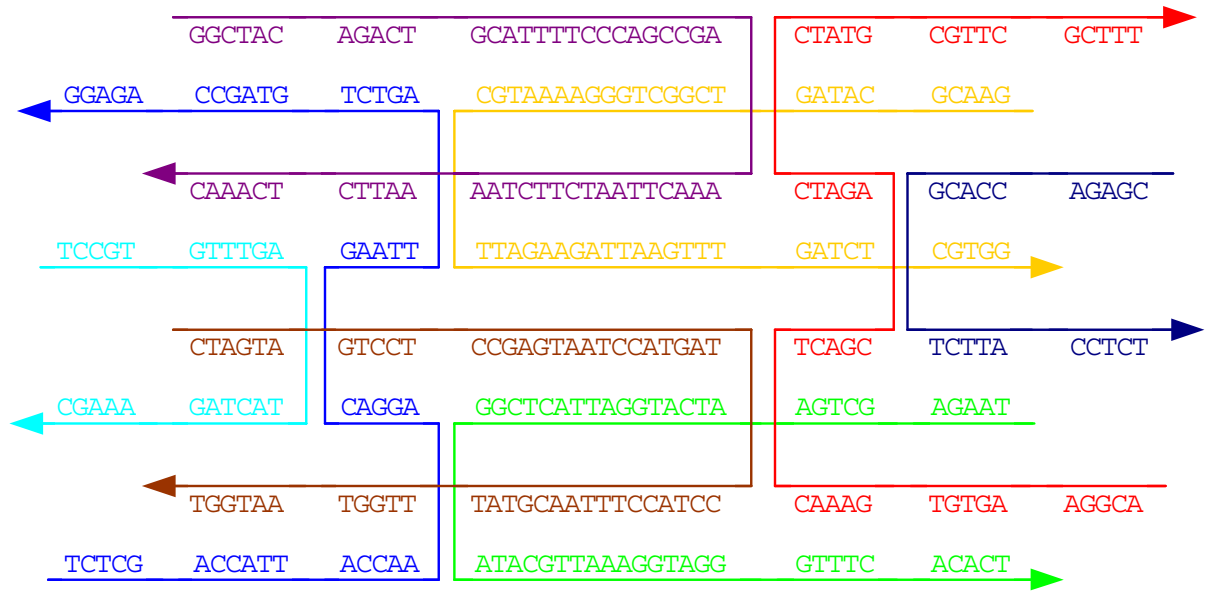

Complexes seen in Figure 3.

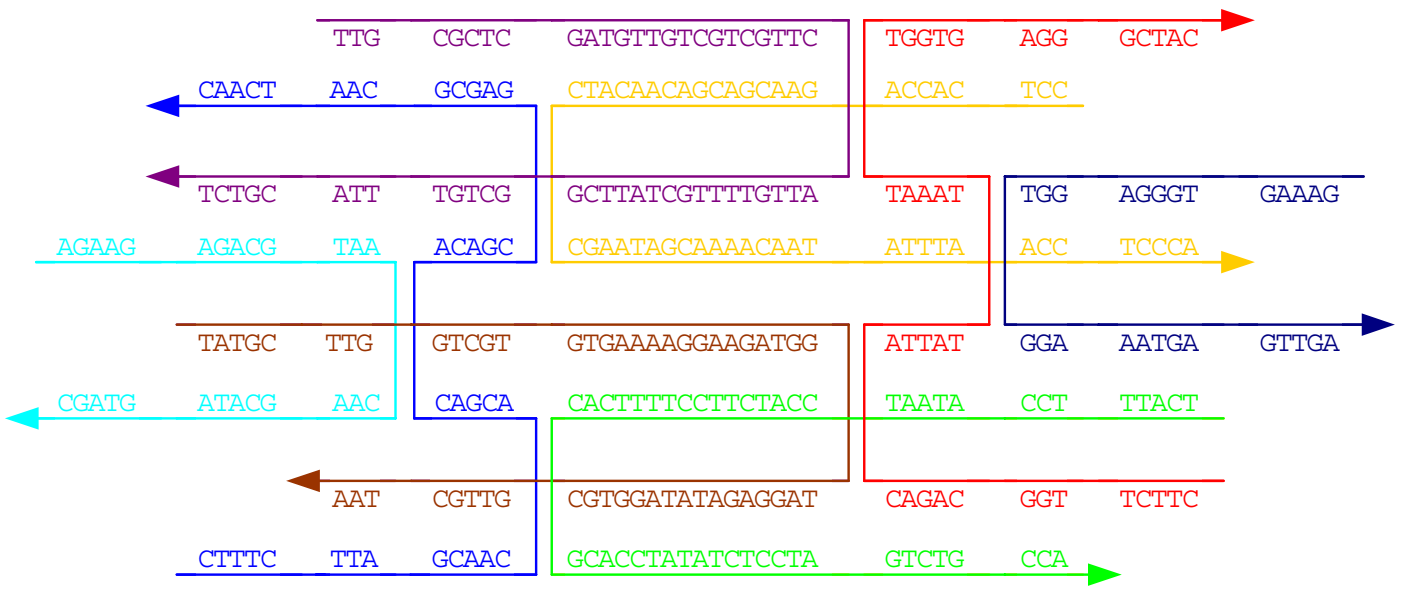

\section{Double-double crossover creation:}

DNA strands were synthesized and PAGE purified by Integrated DNA Technologies. A solution of $0.2 \mu \mathrm{M}$ of each strand was prepared in TAE/ $\mathrm{Mg}^{2+}$ buffer (40 mM Tris-HCl, $\mathrm{pH} 8.0 ; 1 \mathrm{mM}$ EDTA; $12.5 \mathrm{mM}$ Magnesium Acetate). For the complexes in Figure 2, the solution was heated to $90^{\circ} \mathrm{C}$ in an insulated water bath and left to cool to room temperature over three days. When this protocol was used for the alternative DDX complexes, only tubular structures were seen. For the complexes in Figures $3 \mathrm{c}$ and $3 \mathrm{~d}$, the solution was heated to $90^{\circ} \mathrm{C}$ for 5 minutes, then cooled to $25^{\circ} \mathrm{C}$ over the course of 65 minutes, 1 degree per minute, in a GeneAmp polymerase chain reaction $(\mathrm{PCR})$ thermocycler.

\section{AFM Sample Preparation and Imaging}

A $5 \mu \mathrm{l}$ aliquot of the complexes was spotted onto freshly cleaved mica (Ted Pella), left for 30 seconds and then topped with $25 \mu \mathrm{l}$ of $\mathrm{TAE} / \mathrm{Mg}^{2+}$ buffer. Imaging was performed on a Multimode Nanoscope IIIa atomic force microscope (Digital Instruments) in tapping mode, using a fluid cell, J scanner and $200 \mu \mathrm{m}$ cantilevers with $\mathrm{Si}_{3} \mathrm{~N}_{4}$ tips. 